\title{
Differentiating synchronous double primary lung adenocarcinomas from intrapulmonary metastasis by CT features, EGFR mutations and ALK rearrangement status
}

\author{
Xiaoyu Han ${ }^{1,2 \#}$, Jun Fan ${ }^{3 \#}$, Tong Liu ${ }^{1,2}, \mathrm{Na} \mathrm{Li}^{1,2}$, Osamah Alwalid ${ }^{1,2}, \mathrm{Jin} \mathrm{Gu}^{1,2 *}$, Heshui Shi ${ }^{1,2 *} \wedge$ \\ ${ }^{1}$ Department of Radiology, Union Hospital, Tongji Medical College, Huazhong University of Science and Technology, Wuhan, China; ${ }^{2}$ Hubei \\ Province Key Laboratory of Molecular Imaging, Wuhan, China; ${ }^{3}$ Department of Pathology, Union Hospital, Tongji Medical College, Huazhong \\ University of Science and Technology, Wuhan, China \\ Contributions: (I) Conception and design: H Shi, J Fan, X Han; (II) Administrative support: H Shi, J Gu; (III) Provision of study materials or patients: \\ J Fan, X Han; (IV) Collection and assembly of data: X Han, N Li, O Alwalid, J Gu; (V) Data analysis and interpretation: X Han, J Fan, T Liu, N Li, \\ O Alwalid; (VI) Manuscript writing: All authors; (VII) Final approval of manuscript: All authors. \\ \#These authors contributed equally to this work. \\ *These authors contributed equally to this work. \\ Correspondence to: Heshui Shi, MD, PhD. Department of Radiology, Union Hospital, Tongji Medical College, Huazhong University of Science and \\ Technology, Wuhan 430022, China. Email: heshuishi@hust.edu.cn.
}

Background: Differentiating synchronous double primary lung adenocarcinoma (SDPLA) from interpulmonary metastasis (IPM) has significant therapeutic and prognostic implications. This retrospective study aimed to investigate the potential of computed tomography (CT) features and two known oncogenic driver mutations [epidermal growth factor receptor (EGFR) and anaplastic large-cell lymphoma kinase $(\mathrm{ALK})]$ to discriminate synchronous double primary lung adenocarcinoma from one primary pulmonary adenocarcinoma with intrapulmonary metastasis.

Methods: Patients with SDPLA were selected at our hospital, and those with IPM served as the control group. All 60 patients (40 with SDPLA and 20 with IPM) were tested for EGFR mutations and ALK status, and they underwent chest CT prior to any treatment. Independent-sample Student's $t$-test was used for comparisons between two groups of normally distributed variables, and the Chi-square test was used to compare categorical variables.

Results: The discordance rate of EGFR mutations was significantly higher in patients with SDPLA than in patients with IPM (40\% vs. 5\%, $\mathrm{P}<0.001)$. The incidence of ALK-positive status was $15 \%$, and patients with IPM were more likely to be ALK-positive than patients with SDPLA (35\% vs. 5\%, $\mathrm{P}<0.001$ ). Compared to IPM, SDPLA more frequently occurred in different lobes $(\mathrm{P}=0.024)$, presented with less lymphadenopathy $(\mathrm{P}=0.014)$, showed a smaller difference in diameter $(\Delta \mathrm{d})$ between tumors $(\mathrm{P}=0.001)$ and more commonly presented as lobulated tumors $(\mathrm{P}<0.001)$, spiculated masses $(\mathrm{P}<0.001)$, ground-glass opacities $(\mathrm{GGOs})$ $(\mathrm{P}=0.001)$ and air bronchograms $(\mathrm{P}=0.020)$.

Conclusion: Patients with SDPLA showed higher discordance with EGFR mutations and were less frequently ALK-positive than those with IPM. Thus, the CT characteristics are significantly different between SDPLA and IPM.

Keywords: Adenocarcinoma; lung neoplasms; epidermal growth factor receptor (EGFR); X-ray computed tomography

Submitted Nov 03, 2019. Accepted for publication Sep 03, 2020.

doi: $10.21037 /$ jtd-19-3570

View this article at: http://dx.doi.org/10.21037/jtd-19-3570

\footnotetext{
$\wedge$ ORCID: 0000-0001-7644-3711.
} 


\section{Introduction}

In recent decades, lung cancer has become one of the leading causes of cancer-related deaths worldwide. The incidence of multiple primary lung cancers (MPLCs) in reported clinical series ranges from $1 \%$ to $7 \%$ (1). Recently, autopsy studies have more accurately revealed that the incidence of multiple primary tumors in lungs ranges from $3.5 \%$ to $14 \%$ (2). Differentiating synchronous double primary lung adenocarcinoma (SDPLA) from interpulmonary metastasis (IPM) has significant therapeutic and prognostic implications. However, when two tumors sharing common histological features exist simultaneously in the lungs, their classification as metastases or as separate primary tumors becomes challenging. Therefore, exploring more convenient and accurate methods to differentiate two concurrent lung tumors is important.

Criteria for the identification of multiple primary lung tumors were initially published by Martini and Melamed in 1975 (3) and were updated by the American College of Chest Physicians (ACCP) (4). These criteria are mainly based on tumor locations and histological findings, and neither set of criteria incorporates the use of molecular data to distinguish separate primary tumors from metastases. However, some cases do not completely meet these criteria (5). In recent years, studies have tried to identify SDPLA and IPM using molecular genetics and imaging techniques (2,6-9). Many indicators based on molecular genetics exist, such as epidermal growth factor receptor (EGFR)/KRAS mutation status $(2,8)$, p53 mutation $(6,10)$ and array comparative genomic hybridization (aCGH) (9). However, none of these methods have been widely recognized. Recent oncology and pathology practice guidelines recommend testing all locally advanced and metastatic adenocarcinomas for the most commonly targeted genetic test abnormalities, such as EGFR mutations and anaplastic lymphoma kinase (ALK) gene rearrangements. EGFR mutations (sensitizing activating mutations) are associated with tumor sensitivity to EGFR tyrosine kinase inhibitors (gefitinib, erlotinib and afatinib) (11), and crizotinib was the first drug approved for adenocarcinoma harboring ALK rearrangements $(12,13)$. Therefore, EGFR and ALK tests may be helpful for distinguishing patients with SDPLA from those with IPM in a highly cost-effective manner.

Currently, computed tomography (CT) is the best method to detect and characterize pulmonary tumors (14). Some studies have supported the lack of characteristic imaging findings for SDPLA $(10,15)$. However, signs of malignancy (e.g., lobulation, spiculate and pleural indentations) are often found in each MPLC tumor (16), and IPM lesions typically manifest as solid nodules with smooth margins on CT. Therefore, CT may possess the potential to discriminate SDPLA from IPM.

Therefore, we aimed to investigate the potential of two known oncogenic driver mutations (EGFR and ALK) and CT features to discriminate SDPLA from IPM disease. We also identified relationships among EGFR mutation status, clinical features and CT characteristics in patients with SDPLA.

We present the following article in accordance with the STROBE reporting checklist (available at http://dx.doi. org/10.21037/jtd-19-3570).

\section{Methods}

\section{Patients and inclusion criteria}

In total, 1,193 patients evaluated by the multidisciplinary thoracic oncology group between January 2012 and April 2018 at our institution were retrospectively screened. Among them, 40 patients (80 lesions) with two primary tumors, including a synchronous pulmonary tumor diagnosed within 6 months after diagnosis of the index tumor and lung cancer that metastasized to the same lobe (stage IIIb) or different lobes (stage IV), were consecutively screened and included in the control group (or IPM group) until the sample size was achieved (20 patients and 40 lesions). The inclusion criteria were as follows: (I) available pathology reports (including predominant pathological subtypes, lymph node metastasis and pleural invasion) indicating a diagnosis of lung adenocarcinoma; (II) available results for both EGFR mutations and ALK status; and (III) available clinical data, including age, sex, smoking history and tumor node metastasis (TNM) stage. The exclusion criteria were as follows: (I) thin-section CT was not available; (II) preoperative treatment was administered, such as radiation therapy or chemotherapy; and (III) surgery was not performed. An overview of the inclusion and exclusion criteria is presented in a flow chart (Figure 1A).

The criteria for the definitions of SDPLA and IPM were according to comprehensive histologic assessment (CHA) $(17,18)$. SDPLA was histologically indicated as follows: (I) at least one of the multiple lesions was adenocarcinoma in situ (AIS); (II) the major histopathologic subtype was different among multiple lesions; (III) the major histopathologic subtype was similar, but there were differences in other histological subtypes/cytology/stroma; and (IV) the tumor- 


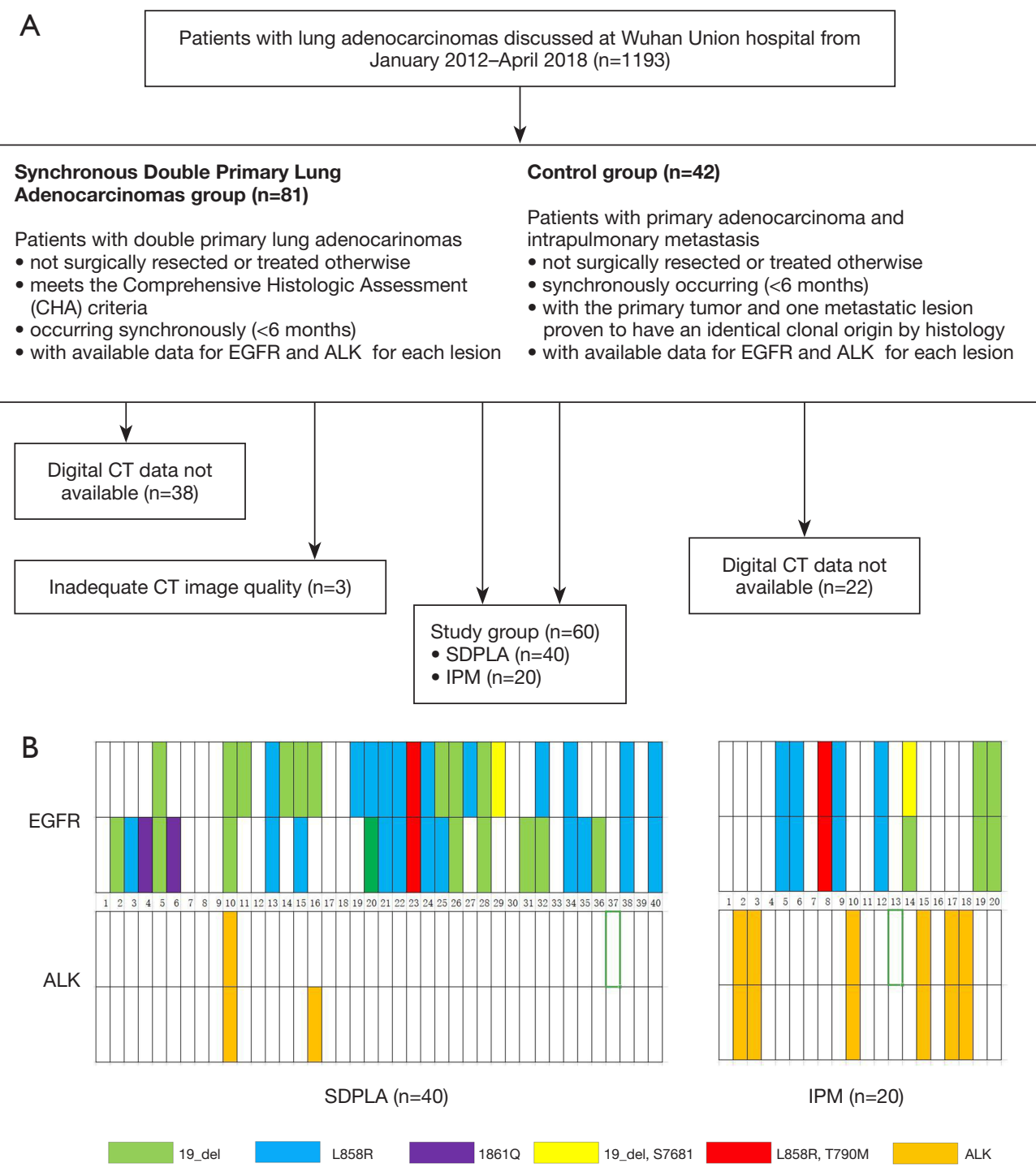

Figure 1 An overview of the inclusion and exclusion criteria (A) as well as molecular testing results (B). SDPLA, synchronous double primary lung adenocarcinoma; IPM, intrapulmonary metastasis; EGFR, epidermal growth factor receptor; ALK, anaplastic large-cell lymphoma kinase.

free interval between cancers was $<2$ years. In addition, IPM was indicated as follows: (I) similar major histopathologic subtype and similar other histological subtypes; and (II) similar major histopathologic subtype and similar growth pattern/architecture.

Patient clinical characteristics, including age, sex, smoking history, histopathology, tumor size, nodal involvement, distant metastasis, and tumor stage, were recorded. Nonsmoking status was defined as lifetime exposure to fewer than 100 cigarettes, and the remaining patients were categorized as ever-smokers. Tumor node metastasis (TNM) staging was based on the IASLC TNM Lung Cancer Staging System ( $8^{\text {th }}$ edition). All CT data were independently reviewed by two experienced radiologists who were blinded to EGFR and ALK status. The medical records of each patient were reviewed retrospectively. This study was approved by the Ethics Committee of Tongii Medical College of Huazhong University of Science and Technology (No. S377). The informed consent was waived by the Ethics Committee for this retrospective study. The study was conducted in accordance with the Declaration of Helsinki (as revised in 2013). 


\section{EGFR mutation analysis}

The overall distribution of EGFR mutations is presented in Figure 1B. EGFR mutations were analyzed according to the principle of the amplified drug resistance mutation system (ARMS). Primary tumors, lymph nodes, distant metastases or pleural effusion specimens were excised, aspirated or biopsied followed by $10 \%$ neutral buffered formalin fixation and paraffin embedding. DNA was extracted from formalinfixed paraffin-embedded tissue sections and analyzed using the Qiagen FFPE Tissue Kit (Netherlands, Roots, NV) according to the manufacturer's instructions. PCR was performed using the Mx3000PtM System (Stratagene, La Jolla, CA, USA) and the EGFR 29 Mutations Detection Kit (Amoy Diagnostics, Xiamen, People's Republic of China), and the results were interpreted according to the manufacturer's instructions.

\section{VENTANA ALK immunobistochemical (IHC) assay}

VENTANA is a fully automated IHC detection method based on the D5F3 monoclonal antibody. The VENTANA IHC assay has been approved by the US FDA and China FDA for the identification of patients with non-small cell lung cancer (NSCLC) who are eligible for treatment with ALK tyrosine kinase inhibitors (TKIs). Formalin-fixed paraffin-embedded tissue sections with a thickness of $4 \mu \mathrm{m}$ were cut according to the manufacturer's instructions and scored using a scoring algorithm. The presence of any percentage of positive tumor cells with strong granular cytoplasmic staining was regarded as ALK positivity, while all other observations were regarded as ALK negativity.

\section{CT image acquisition}

CT was performed at our institution using a multislice spiral CT system (SOMATOM Definition AS +, Siemens Healthineers, Germany). Scanning was performed from the level of the chest inlet to the inferior level of the costophrenic angle. The CT parameters were as follows: detector collimation width, $128 \times 0.6 \mathrm{~mm}$; and tube voltage, $120 \mathrm{kV}$. The tube current was regulated by an automatic exposure control system (CARE Dose 4D). The image thickness and interval were both $5 \mathrm{~mm}$, and the matrix size was $512 \times 512$. Thin-slice reconstruction $(1.5 \mathrm{~mm})$ and image postprocessing were performed after scanning.

Two radiologists with different degrees of experience in interpreting chest CT images independently performed all qualitative image analyses. One was a senior radiologist with 10 years of experience in thoracic imaging, and the other was a fellow with 4 years of experience in the interpretation of CT images. Both analyzed the Digital Imaging and Communications in Medicine (DICOM) images from the CT studies without access to clinical and histological findings but were aware of the presence and sites of the tumors. If their interpretations differed, the senior reader's decision was accepted. Data for each CT scan are presented in Table 1.

\section{Statistical analysis}

Statistical analyses of all data were performed using SPSS software (SPSS 21.0 for Windows, IBM, Chicago, IL, USA). In the two groups, clinical characteristics (age, sex, smoking history and TNM stage) were described on a patient basis. CT findings, EGFR status and ALK status description were described on a lesion basis. Interobserver agreement was assessed by the $k$ coefficient. The normality of the distribution was checked using the KolmogorovSmirnov test. Normally distributed data, non-normally distributed data and categorical variables were expressed as the mean \pm standard deviation, median (interquartile range) and frequency (percentage), respectively. The independentsample Student's $t$ test was used for comparisons of two groups of normally distributed variables, and the Chisquare test was used to compare categorical variables. Pearson correlation analyses were performed for normally distributed variables, and Spearman correlation analyses were performed for non-normally distributed data. A $\mathrm{P}$ value $<0.05$ (two-tailed) was considered statistically significant.

\section{Results}

\section{Clinical characteristics}

In total, 60 eligible patients with synchronous malignancies (40 SDPLA and 20 IPM) were included. The mean age of the SDPLA patients (15 males and 25 females) was $57 \pm 7$ years. Other patient characteristics, including smoking status, are presented in Table 2. No significant differences were found between the two groups in terms of age $(57 \pm 7$ vs. $55 \pm 10$ years, $\mathrm{P}=0.336)$, sex $(\mathrm{P}=1.000)$ or smoking history $(\mathrm{P}=0.541)$. Regarding clinical staging, 19 patients with SDPLA $(47.5 \%)$ presented with stage IIIb or IV disease, 
Table 1 CT features for lung adenocarcinoma

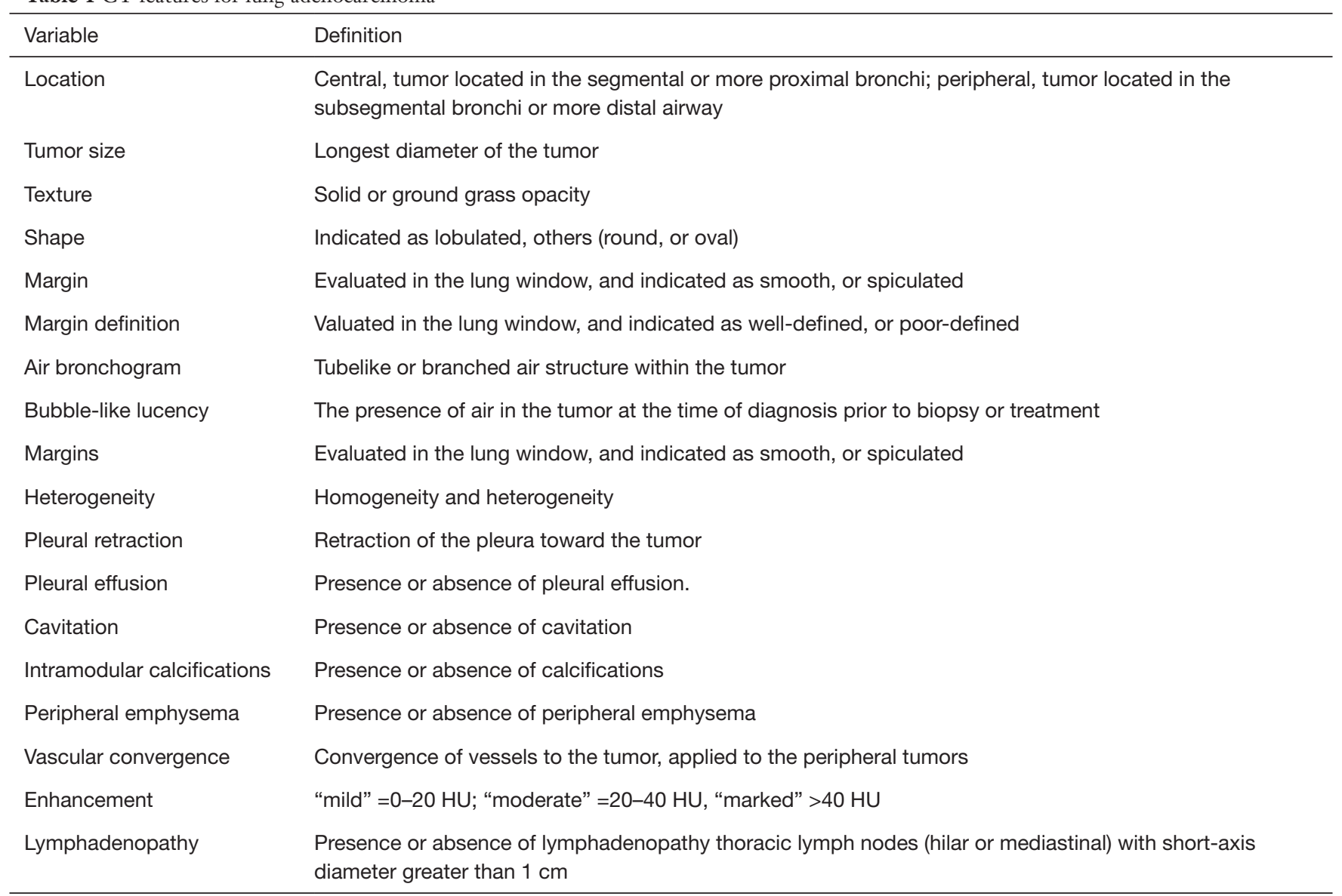

while most patients with IPM (85\%) had an advanced TNM stage (IIIb-IV) (Table 2).

\section{Histopathology, EGFR and ALK results}

Regarding tumor histology, SDPLA tumors were more frequently associated with acinar predominant subtypes (42.5\%) similar to IPM tumors (60\%). When tumors were divided into lepidic predominant adenocarcinomas (adenocarcinoma in situ, minimally invasive adenocarcinoma and lepidic predominant invasive adenocarcinoma) and other subtypes of dominant histological findings (acinar, papillary, micropapillary and solid predominant adenocarcinoma as well as variants of invasive adenocarcinoma), SDPLA tumors were more likely to be lepidic predominant adenocarcinomas than IPM tumors $(25 \%$ vs. $0, \mathrm{P}<0.001)$. Among the 40 cases of SDPLA, 28 patients with SDPLA had at least one tumor exhibiting EGFR mutations (70\%). Among 80 tumors of the 40 patients with SDPLA, 45 lesions showed EGFR mutations $(56.3 \%)$. The incidence of EGFR mutations in this series was $49.2 \%$, whereas patients with SDPLA were more likely to have EGFR mutations than patients with $\operatorname{IPM}(56.3 \%$ vs. 35\%, $\mathrm{P}=0.028)$. In addition, the discordance rate of EGFR mutations was significantly higher in patients with SDPLA than in those with IPM $(40 \%$ vs. $5 \%, \mathrm{P}<0.001)$ (Figure 1B) (Table 3). The incidence of ALK-positive status in our series was $15 \%$, and patients with IPM were more likely to be ALK-positive than patients with SDPLA (35\% vs. $5 \%, \mathrm{P}<0.001)$ (Figure 1B) (Table 3).

\section{Interobserver agreement of CT interpretation}

The concordance between the two observers was almost perfect with $k$ coefficients ranging between 0.65 and 1.0 (Table 4). 
Table 2 Comparison of clinical features between the two groups

\begin{tabular}{|c|c|c|c|}
\hline Variable & $\operatorname{SDPLA}(n=40)$ & IPM (n=20) & $P$ values \\
\hline Age (years) & $57 \pm 7$ & $55 \pm 10$ & 0.336 \\
\hline Sex & & & 1.000 \\
\hline Male & 15 & 8 & \\
\hline Female & 25 & 12 & \\
\hline Smoking history & & & 0.541 \\
\hline Yes & 12 & 4 & \\
\hline No & 28 & 16 & \\
\hline Stage $e^{\&}$ & & & - \\
\hline la & 0 & 0 & \\
\hline $\mathrm{lb}$ & 0 & 0 & \\
\hline Ila & 0 & 0 & \\
\hline $\mathrm{Ilb}$ & 9 & 0 & \\
\hline IIla & 12 & 3 & \\
\hline IIlb & 2 & 2 & \\
\hline IV & 17 & 15 & \\
\hline \multicolumn{4}{|c|}{$\begin{array}{l}\text { \&, according to the IASLC 8th TNM Lung Cancer Staging } \\
\text { System. SDPLA, synchronous double primary lung } \\
\text { adenocarcinoma; IPM, intrapulmonary metastasis; EGFR, } \\
\text { epidermal growth factor receptor; ALK, anaplastic large-cell } \\
\text { lymphoma kinase. }\end{array}$} \\
\hline
\end{tabular}

\section{CT signs}

Compared with IPM, SDPLA was more frequently located in different lobes $(\mathrm{P}=0.024)$, presented less frequently with lymphadenopathy $(\mathrm{P}=0.014)$ and showed a smaller difference $(\Delta \mathrm{d})$ in the largest diameter between tumors $(\mathrm{P}=0.001)$. Tumors in the SDPLA group more frequently presented as GGOs $(\mathrm{P}=0.001)$ (Figure 2), lobulated $(\mathrm{P}<0.001)$ or spiculated $(\mathrm{P}<0.001)$ masses and air bronchograms $(\mathrm{P}=0.020)$ (Figure 3), while tumors in the IPM groups more frequently presented as round/oval solid nodules (Figure 4) with smooth margins on CT images. The incidences of other CT signs were similar between the two groups (Table 5).

\section{Discussion}

The differentiation of SDPLA and IPM is clinically important. However, clinical and imaging criteria for differential diagnosis are not well established. In this study, we identified that SDPLA groups had higher discordance
Table 3 Comparison of pathology and gene status between tumors of two groups

\begin{tabular}{lccc}
\hline Variable & SDPLA $(n=40)$ & IPM $(n=20)$ & P values \\
\hline Histological subtype & & & \\
Lepidic predominant & 20 & 0 & $<0.001^{*}$ \\
Others subtypes & 60 & 40 & \\
Acinar predominant & 34 & 24 & - \\
Papillary predominant & 15 & 12 & - \\
Solid predominant & 7 & 6 & - \\
EGFR & & & $0.028^{\star}$ \\
Mutation & 45 & 14 & \\
Wild-type mutation & 35 & 26 & \\
EGFR mutation & & & $<0.001^{*}$ \\
Identical & 24 & 19 & \\
Discrepancy & 16 & 1 & \\
ALK expression & & & \\
Positive & 4 & 26 & \\
Negative & 76 & 14 & \\
\hline & & &
\end{tabular}

*, $P$ values were based on comparisons between the two groups. SDPLA, synchronous double primary lung adenocarcinoma; IPM, intrapulmonary metastasis; EGFR, epidermal growth factor receptor; ALK, anaplastic large-cell lymphoma kinase.

rates of EGFR mutations and less commonly ALK-positive mutations compared to IPM patients. CT characteristics were found to be significantly different between SDPLA and IPM.

Patients with SDPLA (52.5\%) typically had two earlystage tumors (I-IIIa), while most of those with IPM (85\%) had an advanced TNM stage (IIIb-IV). This finding was consistent with the results of a previous study (7). Moreover, regarding tumor histology, SDPLA tumors were more likely to be lepidic predominant adenocarcinomas than IPM tumors. These findings indicated that patients with SDPLA were at an earlier stage than those with IPM and that their cancer was potentially curable.

TKIs have shown remarkable therapeutic effects and enable prolonged progression-free survival (PFS) in NSCLC patients with EGFR mutations or ALK rearrangements $(12,13)$. These discoveries have led to the recommendation of molecular profiling as the standard of care for advanced NSCLC patients. EGFR mutations have been found in adenocarcinomas in female nonsmokers $(19,20)$, and the 
Table 4 Analysis of inter-reader agreement percent of concordance and kappa of agreement

\begin{tabular}{|c|c|c|c|}
\hline CT features & $\mathrm{N}$ (\% of concordance) & Kappa $(95 \%$ Cl) & Kappa interpretation \\
\hline Size & $154 / 160$ & $0.90(0.86-0.93)$ & Almost perfect \\
\hline location & $156 / 160$ & $0.95(0.94-1.00)$ & Almost perfect \\
\hline Texture & $153 / 160$ & $0.88(0.79-0.96)$ & Almost perfect \\
\hline Margins & $143 / 160$ & $0.73(0.69-0.76)$ & Substantial \\
\hline Vascular convergence & $152 / 160$ & $0.87(0.78-0.93)$ & Almost perfect \\
\hline Air bronchogram & $147 / 160$ & $0.77(0.69-0.84)$ & Substantial \\
\hline Cavitation & $159 / 160$ & $0.98(0.96-1.00)$ & Almost perfect \\
\hline Calcifications & $151 / 160$ & $0.86(0.84-0.89)$ & Almost perfect \\
\hline Enhancement degree & $156 / 160$ & $0.96(0.93-1.00)$ & Almost perfect \\
\hline Lymphadenopathy & $153 / 160$ & $0.89(0.85-0.91)$ & Almost perfect \\
\hline Heterogeneity & $140 / 160$ & $0.68(0.65-0.72)$ & Substantial \\
\hline
\end{tabular}
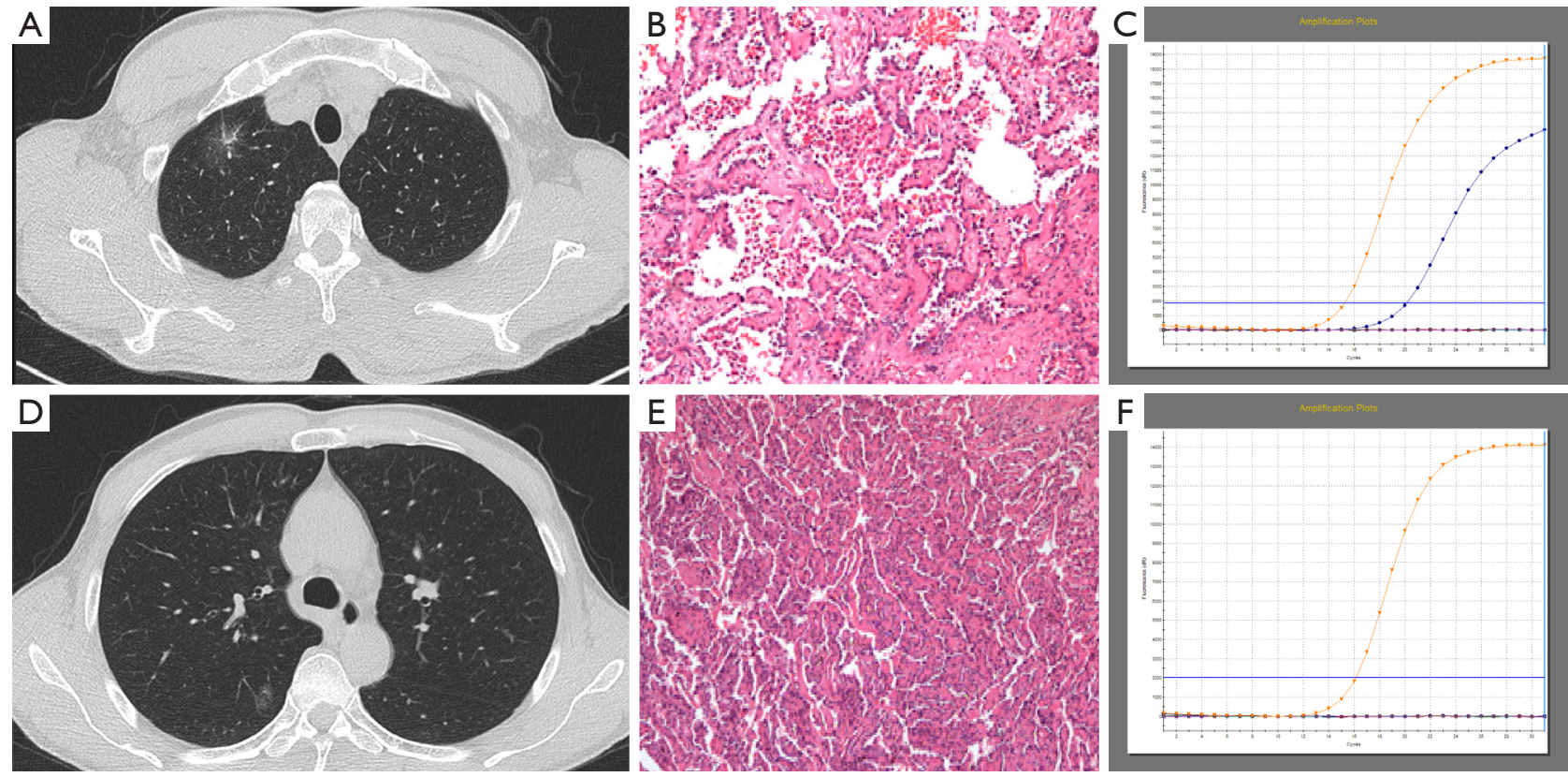

Figure 2 A 61-year-old female with double primary lung adenocarcinomas with one in the right upper lobe (Tumor A, A) and one in the right lower lobe (Tumor B, D). Tumor A appeared as a mixed ground grass opacity (mGGO) with a lobulated border on CT. Tumor B appeared as a pure ground grass opacity (pGGO) on CT. Hematoxylin-eosin staining (B,E) showed different histological types of adenocarcinoma $(\times 100)$, and the ARMS method $(\mathrm{C}, \mathrm{F})$ revealed a 19_del mutation within exon 19 of the epidermal growth factor receptor (EGFR) gene in Tumor A but not in Tumor B. 

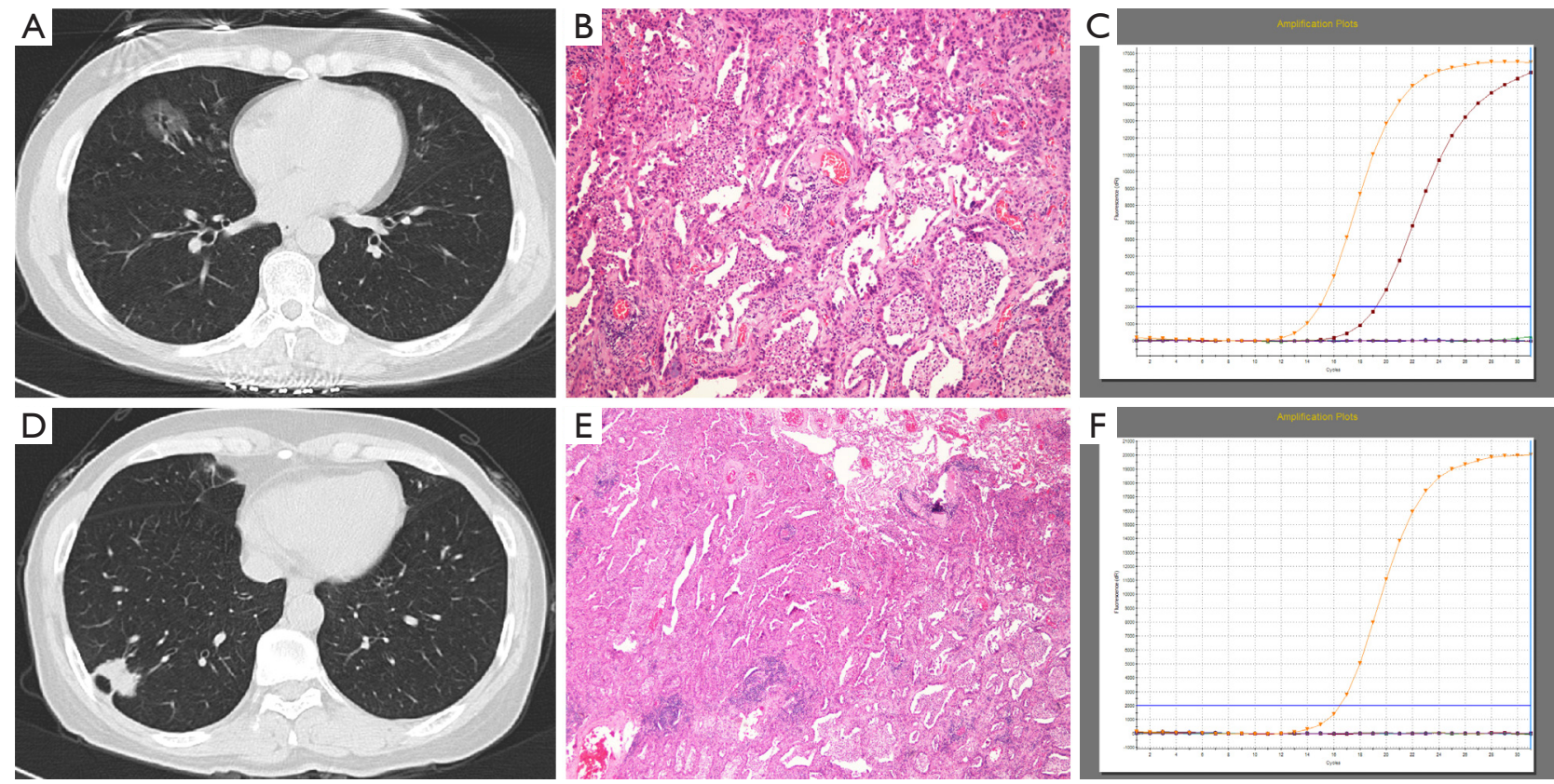

Figure 3 A 53-year-old female with double primary lung adenocarcinomas with one in the right middle lobe (Tumor A, A) and one in the right middle lower lobe (Tumor B, D). Tumor A appeared as a pure ground-glass opacities (GGOs) with air bronchogram. Tumor B appeared as a solid nodule with pleural retraction on CT. Hematoxylin-eosin staining (B,E) showed different histological types of adenocarcinoma $(\times 100)$, and the ARMS method $(\mathrm{C}, \mathrm{F})$ revealed a L858R mutation within exon 21 of the epidermal growth factor receptor (EGFR) gene in Tumor A but not in Tumor B.
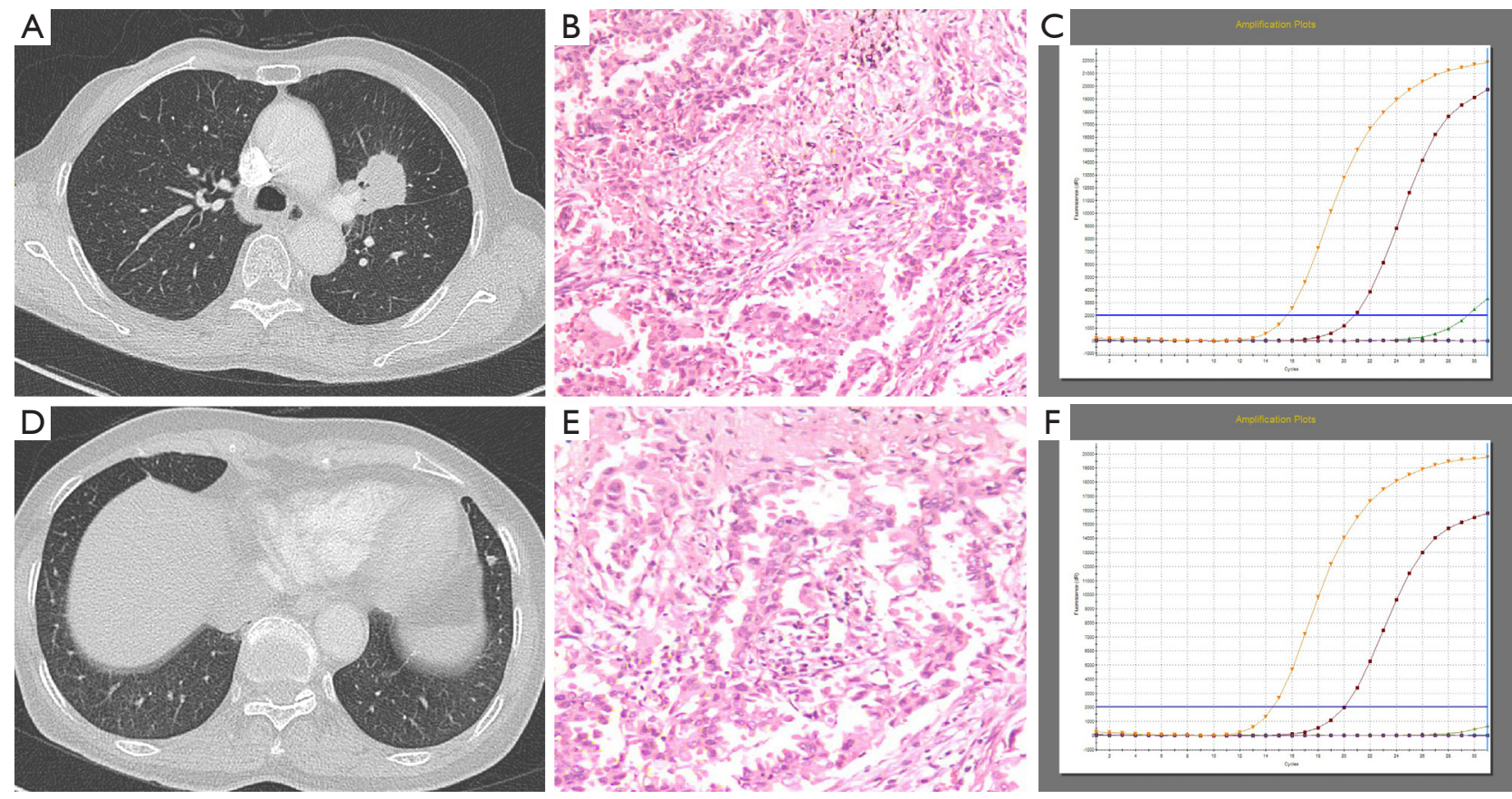

Figure 4 A 73-year-old male with one primary lung adenocarcinoma in the left upper lobe (A) and one metastasis in the left lower lobe of the same histological type $(\mathrm{HE}, \times 200)$ and same epidermal growth factor receptor $(\mathrm{EGFR})$ mutation status. 
Table 5 Comparison of CT features between SDPLA and IPM

\begin{tabular}{|c|c|c|c|}
\hline Characteristic & SDPLA $(n=40)$ & IPM $(n=20)$ & $P$ values \\
\hline Tumor location & & & $0.024^{\star}$ \\
\hline Same lobe & 11 & 12 & \\
\hline Different lobe & 29 & 8 & \\
\hline Size difference ${ }^{\#}$ & $1.1 \pm 0.9$ & $2.4 \pm 1.9$ & $0.001^{*}$ \\
\hline Texture & & & $0.001^{*}$ \\
\hline Solid & 47 & 37 & \\
\hline GGO & 33 & 3 & \\
\hline Shape & & & $<0.001^{\star}$ \\
\hline Lobulated & 63 & 9 & \\
\hline Round/oval & 17 & 31 & \\
\hline Margin & & & $<0.001^{*}$ \\
\hline Smooth & 22 & 26 & \\
\hline Spiculate & 58 & 14 & \\
\hline Margin definition & & & 0.838 \\
\hline Well-defined & 54 & 25 & \\
\hline Poorly-defined & 26 & 15 & \\
\hline Air bronchogram & & & $0.020^{\star}$ \\
\hline Yes & 15 & 1 & \\
\hline No & 65 & 39 & \\
\hline Pleural attachment & & & 0.100 \\
\hline Yes & 31 & 9 & \\
\hline No & 49 & 31 & \\
\hline
\end{tabular}

Table 5 (continued)

mutation rate has been reported to be $27-56 \%$ in Asian patients (19-21). The incidence of EGFR mutations in the present study was $49.2 \%$, whereas SDPLA tumors were more likely to have EGFR (56.3\%) mutations than IPM tumors (35\%). This may be due to ethnic differences in the study population and the diagnostic procedures used. Many studies have found that EGFR gene mutations have a high inconsistency rate (80-92.1\%) in multiple primary NSCLC, especially when the lesion appears as multiple ground-glass nodules $(22,23)$. The inconsistency rate of EGFR mutations in the present study was significantly higher in patients with SDPLA than in patients with IPM. However, the overall EGFR mutation inconsistency rate in SDPLA was $40 \%$, which was much lower than that reported in previous
Table 5 (continued)

\begin{tabular}{|c|c|c|c|}
\hline Characteristic & SDPLA $(n=40)$ & IPM (n=20) & $P$ values \\
\hline Bubble-like lucency & & & 0.138 \\
\hline Yes & 12 & 2 & \\
\hline No & 68 & 38 & \\
\hline Vascular convergence & & & 0.542 \\
\hline Yes & 20 & 8 & \\
\hline No & 60 & 32 & \\
\hline Cavitation & & & 0.605 \\
\hline Yes & 6 & 2 & \\
\hline No & 74 & 38 & \\
\hline Calcifications & & & 1.000 \\
\hline Yes & 4 & 2 & \\
\hline No & 76 & 38 & \\
\hline Lymphadenopathy & & & $0.014^{*}$ \\
\hline Yes & 7 & 10 & \\
\hline No & 33 & 10 & \\
\hline Heterogeneity & & & 0.114 \\
\hline Yes & 28 & 20 & \\
\hline No & 52 & 20 & \\
\hline \multicolumn{4}{|c|}{$\begin{array}{l}\text { *, P values were based on comparisons between the two } \\
\text { groups; \#, the maximum diameter difference }(\Delta d) \text { between } \\
\text { the two lesions. SDPLA, synchronous double primary lung } \\
\text { adenocarcinoma; IPM, intrapulmonary metastasis; GGO, ground } \\
\text { grass opacity. }\end{array}$} \\
\hline
\end{tabular}

studies $(22,23)$. Such a distinction may have been observed because EGFR mutation inconsistency is more common in cases with multiple GGOs, while the incidence of GGOs in our study was low (30\%). One patient in a previous study had a diagnosis of SDPLA based on differences in EGFR mutation status, while the pathological types of the tumors were similar (5). However, it is not reliable to distinguish SDLA from IPM merely on EGFR mutation status because discordance of EGFR mutations between primary tumors and their corresponding metastases in pulmonary adenocarcinoma has been observed $(24,25)$.

ALK is a tyrosine kinase receptor that shows a dramatic response and prolongs PFS with ALK TKI treatment in NSCLC patients (13). ALK rearrangements have a low 
incidence (3\% to $5 \%$ of NSCLC cases) (26) and occur more frequently in young, never-smoker patients with clinically advanced lung adenocarcinoma (27). Studies have reported that ALK-positive tumors are more invasive NSCLCs and are associated with a larger volume, lymph node metastasis or distant metastasis, indicating poor prognosis $(28,29)$. In our data, the rate of ALK-positive SDPLA was 5\% similar to the rate of single pulmonary adenocarcinoma (26). Therefore, the EGFR mutation status of SDPLA in the same patient may be independent without mutual interference. However, patients with IPM had a much higher ALK-positive rate (35\%). As patients with IPM (85\%) typically had a more advanced TNM stage (IIIbIV) in our study, ALK positivity may be related to advanced tumor status and metastatic status.

Regarding CT signs in the present study, SDPLA tumors occurred more frequently in different lung lobes than IPM tumors, which was consistent with previous studies (9). This phenomenon is related to the fact that metastasis commonly occur sin the same lobe and ipsilateral to the primary lesion. Some studies have supported the lack of characteristic imaging findings in SDPLA $(10,15)$. Dijkman et al. (7) found that a definite diagnosis based on CT morphological features can be made in only $50 \%$ of patients $(8 / 16)$ in the second primary group. However, in the present study, SDPLA more commonly presented as GGOs, while IPM more frequently presented as solid nodules. This might be related to the fact that GGOs are considered multiple primary lung cancers and are detected at an early stage $(30,31)$. Li et al. (16) reported that multiple synchronous lesions commonly present as single or multiple subsolid nodules. Moreover, as mentioned earlier, SDPLA patients were more likely to have EGFR (56.3\%) mutations than IPM patients (35\%), while patients with IPM had a much higher rate (35\%) than SDPLA (5\%). Previous data (21) have shown that the occurrence of GGOs is significantly associated with EGFR mutations and that the presence of solid nodules is one of the distinctive CT features of ALK rearrangement lung adenocarcinomas (32). Therefore, these results may also account for the potential association between EGFR and GGO as well as the association between ALK and solid nodules. In the present study, SDPLA more frequently presented as lobulated, spiculated and air bronchograms than IPM, which was consistent with previous studies (16), while IPM more frequently presented as round/oval solid nodules with smooth margins on CT images. These findings indicated that each SDPLA lesion tended to have the malignant characteristics of primary lung cancer. Moreover, IPM patients showed lymph node enlargement more frequently than SDPLA patients, which was confirmed as lymph node metastasis by surgery and was associated with the higher tumor stage of IPM.

Our study had some limitations. (I) This study was performed at a single center with a small series of cases; thus, multicenter studies with large sample sizes are needed to verify our conclusions. (II) This study was a retrospective analysis, and although we strictly used double-blind methods to record CT signs, EGFR gene mutations and ALK results, selection bias was inevitable. (III) We classified the tumors by histological features, which were not verified by molecular biology. (IV) In this study, the prognosis between the two groups was not provided because the cases were collected from 2012 to 2018 and the follow-up time was insufficient.

In conclusion, CT features, EGFR mutations and ALK status may help differentiate SDPLA from IPM. Our preliminary results encourage the establishment of comprehensive diagnostic criteria, including clinical characteristics, histological features, CT images and gene analysis in further study.

\section{Acknowledgments}

Funding: None.

\section{Footnote}

Reporting Checklist: The authors have completed the STROBE reporting checklist. Available at http://dx.doi. org/10.21037/jtd-19-3570

Data Sharing Statement: Available at http://dx.doi. org/10.21037/jtd-19-3570

Conflicts of Interest: All authors have completed the ICMJE uniform disclosure form (available at http://dx.doi. org/10.21037/jtd-19-3570). The authors have no conflicts of interest to declare.

Ethical Statement: The authors are accountable for all aspects of the work, ensuring that questions related to the accuracy or integrity of any part of the work are appropriately investigated and resolved. This study was approved by Ethics Committee of Tongji Medical College of Huazhong University of Science and Technology (No. S377). The informed consent was waived by the Ethics 
Committee for this retrospective study. The study was conducted in accordance with the Declaration of Helsinki (as revised in 2013).

Open Access Statement: This is an Open Access article distributed in accordance with the Creative Commons Attribution-NonCommercial-NoDerivs 4.0 International License (CC BY-NC-ND 4.0), which permits the noncommercial replication and distribution of the article with the strict proviso that no changes or edits are made and the original work is properly cited (including links to both the formal publication through the relevant DOI and the license). See: https://creativecommons.org/licenses/by-nc-nd/4.0/.

\section{References}

1. Ferguson MK, DeMeester TR, DesLauriers J, et al. Diagnosis and management of synchronous lung cancers. J Thorac Cardiovasc Surg 1985;89:378-85.

2. Chang YL, Wu CT, Lin SC, et al. Clonality and prognostic implications of $\mathrm{p} 53$ and epidermal growth factor receptor somatic aberrations in multiple primary lung cancers. Clin Cancer Res 2007;13:52-8.

3. Martini N, Melamed MR. Multiple primary lung cancers. J Thorac Cardiovasc Surg 1975;70:606-12.

4. Shen KR, Meyers BF, Larner JM, et al. Special Treatment Issues in Lung Cancer: ACCP EvidenceBased Clinical Practice Guidelines (2nd Edition). Chest 2007;132:290S-305S.

5. Takuwa T, Tanaka F, Yoneda K, et al. Diagnosis of synchronous primary lung adenocarcinomas based on epidermal growth factor (EGFR) gene status: A case report. Lung Cancer 2010;68:498-500.

6. van Rens MT, Eijken EJ, Elbers JR, et al. p53 mutation analysis for definite diagnosis of multiple primary lung carcinoma. Cancer 2002;94:188-96.

7. Dijkman BG, Schuurbiers OC, Vriens D, et al. The role of (18)F-FDG PET in the differentiation between lung metastases and synchronous second primary lung tumours. Eur J Nucl Med Mol Imaging 2010;37:2037-47.

8. Girard N, Deshpande C, Azzoli CG, et al. Use of epidermal growth factor receptor/Kirsten rat sarcoma 2 viral oncogene homolog mutation testing to define clonal relationships among multiple lung adenocarcinomas: comparison with clinical guidelines. Chest 2010;137:46-52.

9. Arai J, Tsuchiya T, Oikawa M, et al. Clinical and molecular analysis of synchronous double lung cancers. Lung Cancer 2012;77:281-7.
10. Matsuzoe D. Discrimination of double primary lung cancer from intrapulmonary metastasis by $\mathrm{p} 53$ gene mutation. Br J Cancer 1999;79:1549-52.

11. Jorge SEDC, Kobayashi SS, Costa DB. Epidermal growth factor receptor (EGFR) mutations in lung cancer: preclinical and clinical data. Braz J Med Biol Res 2014;47:929-39.

12. Mok TS, Wu YL, Thongprasert S, et al. Gefitinib or carboplatin-paclitaxel in pulmonary adenocarcinoma. $\mathrm{N}$ Engl J Med. 2009;361:947-57.

13. Solomon BJ, Mok T, Kim DW, et al. First-line crizotinib versus chemotherapy in ALK-positive lung cancer. $\mathrm{N}$ Engl J Med 2014;371:2167-77.

14. Ren YY, Li YC, Wu HB, et al. Combined use of thinsection CT and (18)F-FDG PET/CT for characterization of solitary pulmonary nodules. Journal of Southern Medical University 2017;37:283.

15. Matsuzoe D, Hideshima T, Ohshima K, et al. Discrimination of double primary lung cancer from intrapulmonary metastasis by $\mathrm{p} 53$ gene mutation. $\mathrm{Br} \mathrm{J}$ Cancer 1999;79:1549-52.

16. Li J, Yang X, Xia T, et al. Stage I synchronous multiple primary non-small cell lung cancer: CT findings and the effect of TNM staging with the 7th and 8th editions on prognosis. J Thorac Dis 2017;9:5335-44.

17. Girard N, Deshpande C, Lau C, et al. Comprehensive Histologic Assessment Helps to Differentiate Multiple Lung Primary Nonsmall Cell Carcinomas From Metastases. Am J Surg Pathol 2009;33:1752-64.

18. Detterbeck FC, Franklin WA, Nicholson AG, et al. The IASLC Lung Cancer Staging Project: Background Data and Proposed Criteria to Distinguish Separate Primary Lung Cancers from Metastatic Foci in Patients with Two Lung Tumors in the Forthcoming Eighth Edition of the TNM Classification for Lung Cancer. J Thorac Oncol 2016;11:651-65.

19. Maemondo M, Inoue A, Kobayashi K, et al. Gefitinib or chemotherapy for non-small-cell lung cancer with mutated EGFR. N Engl J Med 2010;362:2380-8.

20. Paez JG, Jänne PA, Lee JC, et al. EGFR mutations in lung cancer: correlation with clinical response to gefitinib therapy. Science 2004;304:1497-500.

21. Liu Y, Kim J, Qu F, et al. CT Features Associated with Epidermal Growth Factor Receptor Mutation Status in Patients with Lung Adenocarcinoma. Radiology 2016;280:271-80.

22. Wu C, Zhao C, Yang Y, et al. High Discrepancy of Driver Mutations in Patients with NSCLC and Synchronous 
Multiple Lung Ground-Glass Nodules. J Thorac Oncol 2015;10:778-83.

23. Liu M, He WX, Song N, et al. Discrepancy of epidermal growth factor receptor mutation in lung adenocarcinoma presenting as multiple ground-glass opacities. Eur J Cardiothorac Surg 2016;50:909-13.

24. Munfus-McCray D, Harada S, Adams C, et al. EGFR and KRAS mutations in metastatic lung adenocarcinomas. Hum Pathol 2011;42:1447-53.

25. Han HS, Eom DW, Kim JH, et al. EGFR mutation status in primary lung adenocarcinomas and corresponding metastatic lesions: discordance in pleural metastases. Clin Lung Cancer 2011;12:380-6.

26. Rikova K, Guo A, Zeng Q, et al. Global Survey of Phosphotyrosine Signaling Identifies Oncogenic Kinases in Lung Cancer. Cell 2007;131:1190-203.

27. Dacic S. Molecular genetic testing for lung adenocarcinomas: a practical approach to clinically relevant mutations and translocations. J Clin Pathol 2013;66:870-4.

28. Lv Z, Fan J, Xu J, et al. Value of (18)F-FDG PET/CT for predicting EGFR mutations and positive ALK expression in patients with non-small cell lung cancer: a retrospective analysis of 849 Chinese patients. Eur J Nucl Med Mol Imaging 2018;45:735-50.

29. Halpenny DF, Riely GJ, Hayes S, et al. Are there imaging characteristics associated with lung adenocarcinomas harboring ALK rearrangements? Lung Cancer 2014;86:190-4.

30. Naidich DP, Bankier AA, MacMahon H, et al. Recommendations for the management of subsolid pulmonary nodules detected at CT: a statement from the Fleischner Society. Radiology 2013;266:304-17.

31. Detterbeck FC, Marom EM, Arenberg DA, et al. The IASLC Lung Cancer Staging Project: Background Data and Proposals for the Application of TNM Staging Rules to Lung Cancer Presenting as Multiple Nodules with Ground Glass or Lepidic Features or a Pneumonic Type of Involvement in the Forthcoming Eighth Edition of the TNM Classification. J Thorac Oncol 2016;11:666-80.

32. Kim TH, Woo S, Yoon SH, et al. CT Characteristics of Non-Small Cell Lung Cancer With Anaplastic Lymphoma Kinase Rearrangement: A Systematic Review and MetaAnalysis. AJR Am J Roentgenol 2019;213:1059-72.
Cite this article as: Han X, Fan J, Liu T, Li N, Alwalid O, Gu J, Shi H. Differentiating synchronous double primary lung adenocarcinomas from intrapulmonary metastasis by CT features, EGFR mutations and ALK rearrangement status. J Thorac Dis 2020;12(10):5505-5516. doi: 10.21037/jtd-19-3570 